\title{
Correction to: The BR1 Scheme is Stable for the Compressible Navier-Stokes Equations
}

\author{
Gregor J. Gassner ${ }^{1}$ • Andrew R. Winters ${ }^{1}$. \\ Florian J. Hindenlang ${ }^{2}$. David A. Kopriva ${ }^{3}$
}

Published online: 18 June 2018

(C) Springer Science+Business Media, LLC, part of Springer Nature 2018

\section{Correction To: J Sci Comput https://doi.org/10.1007/s10915-018-0702-1}

The original version of this article unfortunately contained an error. The authors would like to correct the error with this erratum.

The open-source code FLUXO implements the entropy stable discontinuous Galerkin scheme with Legendre-Gauss-Lobatto collocation (DGSEM) on curved unstructured hexahedral grids for compressible Navier-Stokes equations (NSE) [3] and is available at github.com/project-fluxo. Numerical investigations show that it is robust in comparison with the standard DGSEM (even in comparison with ad hoc stabilisation techniques such as polynomial de-aliasing) for under-resolved compressible turbulence simulations, e.g. [1].

To further highlight the remarkable robustness of the entropy stable form of the DGSEM, we add the discussion of a numerical simulation of the Taylor-Green vortex with a Reynolds number of $R e=1600$ (see, e.g. [1,2] for details of the set-up). We intentionally stress the numerical experiment by choosing numerical fluxes without added artificial stabilisation terms. That is, we use the BR1 scheme for the viscous terms, and we use only the entropy conserving (EC) flux at the interface for the Euler terms, without adding any Riemann solver type dissipation. To further stress the method at high order and to highlight its enhanced robustness, we intentionally under-resolve the physics of the vortex-dominated flow.

A common challenging benchmark resolution is $64^{3}$ degrees of freedom. We choose a high polynomial degree of $N=7$ (eight GLL nodes in each direction inside the element) and $8^{3}$ elements. If we use the standard DGSEM without the entropy stable modifications,

The original article can be found online at https://doi.org/10.1007/s10915-018-0702-1.

Gregor J. Gassner

ggassner@math.uni-koeln.de

1 Mathematical Institute, University of Cologne, Cologne, Germany

2 Max Planck Institute for Plasma Physics, Boltzmannstraße 2, 85748 Garching, Germany

3 Department of Mathematics, The Florida State University, Tallahassee, FL 32306, USA 


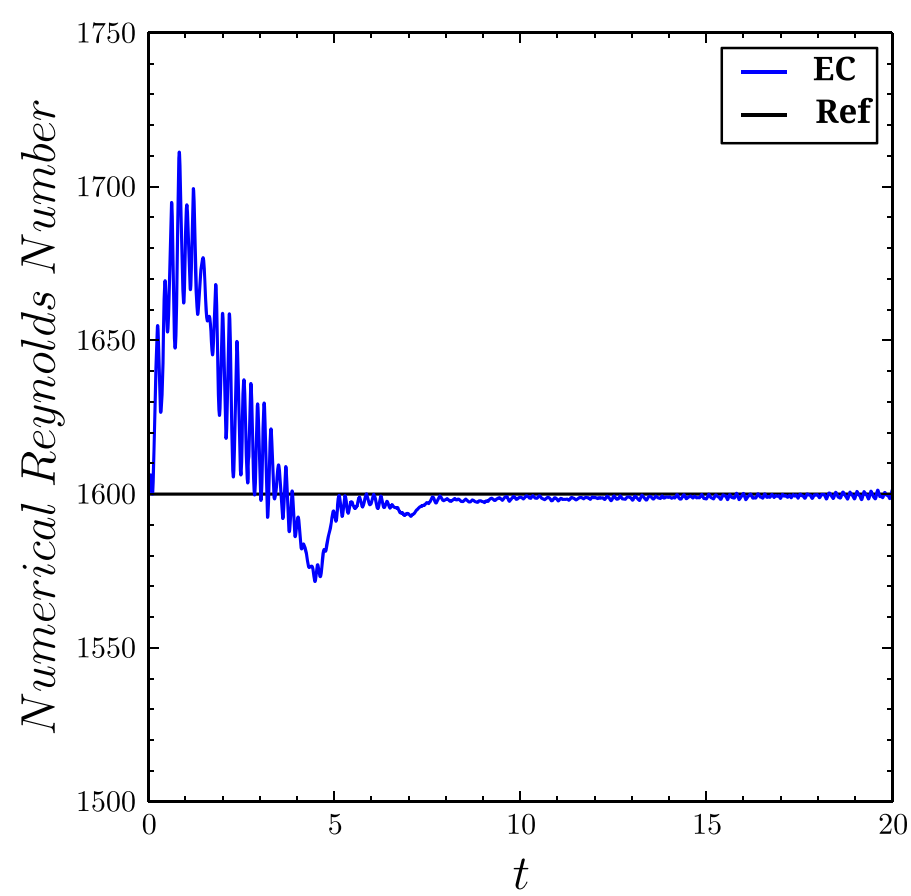

Fig. 1 Numerical simulation of the Taylor-Green vortex with Mach number $M=0.1$ and Reynolds number $R e=1600$ showing temporal evolution of the numerical Reynolds number in comparison with the physical value. The DG discretization uses $8^{3}$ elements and a polynomial degree of $N=7$, resulting in $64^{3}$ degrees of freedom. The BR1 method is used for the viscous terms and the EC flux without additional dissipation is used for the Euler fluxes at the interfaces, making the scheme virtually dissipation-free yet stable. The standard DGSEM immediately crashes for this test case

the simulation immediately crashes, even when using Riemann solvers with added dissipation at the interface, see, e.g. [2]. However, when using the entropy stable approximation even without added numerical dissipation at the element interfaces, the simulation no longer crashes.

In addition, the numerical scheme is virtually dissipation-free without element interface stabilization terms. To see this, we choose a Mach number of $M=0.1$ for the Taylor-Green vortex to allow for comparisons with the incompressible NSE. For incompressible flows, it is possible to relate the enstrophy (ens), the kinetic energy dissipation rate (diss) and the Reynolds number. Using this relationship, we estimate the numerical Reynolds number by computing the ratio of the discrete total enstrophy and the discrete total kinetic energy dissipation rate, i.e. $\operatorname{Re}_{n u m}(t) \approx 2 \frac{e n s(t)}{\operatorname{diss}(t)}$. The result is presented in Fig. 1. As a reference, the physical Reynolds number $R e=1600$ is plotted as well. It is remarkable that for the fully developed flow at later times the numerical Reynolds number approaches the physical Reynolds number, which confirms the two properties proven in the paper: (i) the method is virtually dissipation free; (ii) it is still stable when the standard scheme crashes.

Acknowledgements This work was supported by a grant from the Simons Foundation (\#426393, David Kopriva). G.G. has been supported by the European Research Council (ERC) under the European Union's Eights Framework Program Horizon 2020 with the research project Extreme, ERC Grant Agreement No. 714487. 


\section{References}

1. Gassner, G.J., Winters, A.R., Kopriva, D.A.: Split form nodal discontinuous Galerkin schemes with summation-by-parts property for the compressible Euler equations. J. Comput. Phys. 327, 39-66 (2016)

2. Gassner, G.J., Beck, A.D.: On the accuracy of high-order discretizations for underresolved turbulence simulations. Theoret. Comput. Fluid Dyn. 27(3-4), 221-237 (2013)

3. Gassner, G.J., Winters, A.R., Hindenlang, F.J., Kopriva, D.A.: The BR1 scheme is stable for the compressible Navier-Stokes equations. J. Sci. Comput. (2018) 